\title{
EIGENVALUES OF COXETER TRANSFORMATIONS AND THE GELFAND-KIRILLOV DIMENSION OF THE PREPROJECTIVE ALGEBRAS
}

\author{
VLASTIMIL DLAB ${ }^{1}$ AND CLAUS MICHAEL RINGEL
}

\begin{abstract}
The spectral radius of a Coxeter transformation is shown to be an eigenvalue which can be expressed in terms of lengths of certain positive roots of the corresponding valued graph. This result is used to determine the Gelfand-Kirillov dimension of the preprojective algebras: This dimension is equal to 0,1 or $\infty$ according to whether the underlying graph is Dynkin, Euclidean or otherwise.
\end{abstract}

Let $C=\left(c_{i j}\right)$ be an $n \times n$ Cartan matrix, that is $c_{i i}=2$ for $1<i \leqslant n, c_{i j} \neq 0$ if and only if $c_{j i} \neq 0$, and $c_{i j} \leqslant 0$ is integral for all $i \neq j$. Throughout the paper, $C$ is assumed to be indecomposable and its graph not to be Dynkin. Recall that the associated (in general, not symmetrizable) valued graph is given by $n$ vertices and edges $i \bullet j$ with valuation $\left(-c_{i j},-c_{j i}\right)$ for $i \neq j$ and $c_{i j} \neq 0$ (and with no loops); see [3].

Definition. The reflections $r_{s} \in \mathrm{GL}\left(\mathrm{C}^{n}\right), 1<s<n$, are defined by $r_{s} \mathbf{x}=\mathbf{y}=$ $\left(y_{i}\right)$, where $y_{i}=x_{i}$ for $i \neq s$ and $y_{s}=-x_{s}-\Sigma_{i} c_{i s} x_{i}$. The element $c=r_{n} \cdots r_{2} r_{1}$ is called a Coxeter transformation.

Note that with a $c$, also $c^{-1}$ is a Coxeter transformation (corresponding to a Cartan matrix obtained from $C$ by a permutation of rows and columns). Recall that the spectral radius of a linear transformation $c$ is the maximal $|\lambda|$ of all eigenvalues $\lambda$ of $c$. The degree $d(\lambda)$ of an eigenvalue $\lambda$ of $c$ is the maximal size of the Jordan $\lambda$-blocks of $c$.

Proposition 1. The spectral radius $\rho$ of $c$ is an eigenvalue of $c$ and $d(\rho)>d(\lambda)$ for all eigenvalues $\lambda$ of $c$ with $|\lambda|=\rho$.

The proof of Proposition 1 follows from Theorem 3.1 of J. S. Vandergraft [8], as soon as we construct a (closed) cone with nonempty interior in $\mathbf{R}^{n}$ which is mapped by $c$ into itself. In fact, we shall consider $c^{-1}$ instead of $c$. The authors are indebted to L. Elsner for helpful discussions concerning eigenvalues of linear transformations.

REMARK. Applying Proposition 1 to $c^{-1}$, we conclude that there is an eigenvalue $1 / \rho^{\prime}$ of $c$ with the least absolute value and $d\left(1 / \rho^{\prime}\right)>d(\lambda)$ for all eigenvalues $\lambda$ of $c$

Received by the editors, September 24, 1980; presented to the Society in San Francisco, January 7-11, 1981.

1980 Mathematics Subject Classification. Primary 15A18, 15A48, 16A46, 16A64, 17B20, 20 F05.

Key words and phrases. Cartan matrix, valued graph, Dynkin diagram, Euclidean diagram, positive root, Coxeter transformation, eigenvalue, spectral radius, Gelfand-Kirillov dimension, preprojective algebra, finite, tame and wild representation types.

1 The first author wishes to acknowledge support of NSERC under Grant No. A-7257. 
with $|\lambda|=1 / \rho^{\prime}$. If the Cartan matrix is symmetrizable, then $\rho^{\prime}=\rho$. For, then a quadratic form is defined which is $c$-invariant. For nonsymmetric Cartan matrices, in general $\rho^{\prime} \neq \rho$. If $C$ defines a Euclidean graph, then $\rho^{\prime}=\rho=1$ [3] and therefore all eigenvalues lie on the unit circle. This is, of course, also trivially true when $C$ defines a Dynkin graph (for, $c$ is of finite order). In the case that $C$ defines a tree, sharper results on the eigenvalues of the corresponding Coxeter transformations were obtained by N. A' Campo [1] and V. F. Subbotin and R. B. Stekolščik [7]; however, they do not generalize to the general situation of nonsymmetrizable matrices.

Define the preprojective vectors (or "preprojective roots") $\mathrm{p}(s, t), 1<s<n$, $t>0$, of $C$ by

$$
\mathrm{p}(s, t)=c^{-t} r_{1} r_{2} \cdots r_{s-1} \mathrm{~s},
$$

where $s=\left(\delta_{i s}\right)_{i}$. For $\mathbf{x}=\left(x_{i}\right) \in \mathbf{C}^{n}$, we write $|\mathbf{x}|=\Sigma_{i}\left|x_{i}\right|$.

The fact that the graph defined by $C$ is not Dynkin is equivalent to the statement that all $\mathrm{p}(s, t)$ are distinct. This, in turn, is equivalent to the statement that all $\mathrm{p}(s, t)$ are positive; see [3].

Now, define the (preprojective) closed cone $K$ as the closed convex hull of all $\mathrm{p}(s, t)$. Clearly, $c^{-1} K \subseteq K$. Moreover, since the projective vectors $\{\mathrm{p}(s, 0) \mid 1<s<$ $n$ \} form a basis of $\mathbf{C}^{n}$, the interior $K^{0}$ of $K$ is nonempty.

Proposition 2. For any $\mathrm{p}=\mathrm{p}(s, t)$, we have

$$
\rho=\lim _{m \rightarrow \infty} \sqrt[m]{\left|c^{-m} \mathbf{p}\right|} .
$$

REMARK. The limit

$$
\lim _{m \rightarrow \infty} \sqrt[m]{\left|c^{-m} \mathbf{x}\right|}
$$

clearly measures the growth of the vector $\mathbf{x}$ with respect to $c$ and can be called the "growth number" of $\mathbf{x}$; or, if $\mathbf{x}=\operatorname{dim} X$ for some module $X$, the growth number of $X$. (In general, one may consider $\operatorname{dim}(\operatorname{Tr} D)^{m} X$ instead of $c^{-m} \operatorname{dim} X$ and call

$$
\varlimsup \sqrt[m]{\left|\operatorname{dim}(\operatorname{Tr} D)^{m} X\right|}
$$

the growth number of $X$ [7].)

Corollary. All preprojective vectors have equal growth number. If the graph defined by $C$ is Euclidean, this number is equal to 1. If the graph is neither Dynkin nor Euclidean, it is greater than 1.

Proof of Proposition 2. Let $\mathbf{e}_{i j}, 1<i<q, 1<j<n_{i}$, be a Jordan basis of $\mathbf{C}^{n}$ with respect to which $c^{-1}$ is the product of $q$ Jordan $n_{i} \times n_{i}$ matrices of the form

$$
\left[\begin{array}{ccccccc}
\lambda_{i} & \lambda_{i} & & & & & \\
& \lambda_{i} & \lambda_{i} & & & 0 & \\
& & \cdot & \cdot & & & \\
& & & \cdot & \cdot & \cdot & \\
& 0 & & & & \lambda_{i} & \lambda_{i} \\
& & & & & & \lambda_{i}
\end{array}\right] ;
$$


moreover, in view of Proposition 1, we assume that $\rho=\lambda_{1}>\left|\lambda_{i}\right|$ for all eigenvalues $\lambda_{i}, 1 \leqslant i \leqslant q$. Thus

$$
c^{-1} \mathbf{e}_{i, j}=\lambda_{i}\left(\mathbf{e}_{i, j}+\mathbf{e}_{i, j-1}\right)
$$

here $\mathbf{e}_{i, r}=\mathbf{0}$ for $r \leqslant 0$. Let $\mathbf{p}=\sum_{i, j} \alpha_{i, j} \mathbf{e}_{i, j}$; consequently,

$$
c^{-m} \mathbf{p}=\sum_{i, j} \alpha_{i, j} \lambda_{i}^{m}\left[\mathbf{e}_{i, j}+\left(\begin{array}{c}
m \\
1
\end{array}\right) \mathbf{e}_{i, j-1}+\cdots+\left(\begin{array}{c}
m \\
s
\end{array}\right) \mathbf{e}_{i, j-s}+\cdots\right] .
$$

Now, take $d=\max d\left(\lambda_{i}\right)$ and $s_{m}=\left(\begin{array}{c}m \\ d\end{array}\right)$; thus, for large $m, 1 / s_{m} \cdot\left(\begin{array}{c}m \\ s\end{array}\right) \leqslant 1$ for all $\mathbf{e}_{i, j-s} \neq \mathbf{0}$. Consequently, since

$$
\begin{aligned}
& \lim _{m \rightarrow \infty} \sqrt[m]{\operatorname{mim}} \sqrt[m]{s_{m}}=1=\lambda_{1} \lim _{m \rightarrow \infty} \sqrt[m]{\left|c^{-m} \mathbf{p}\right| / s_{m} \lambda_{1}^{m}} \\
&=\lambda_{1} \lim _{m \rightarrow \infty} \sqrt[m]{\sum_{i, j}\left|\alpha_{i, j}\right|\left|\lambda_{i} / \lambda_{1}\right|^{m} d}=\lambda_{1}=\rho .
\end{aligned}
$$

As an application of the previous results, we are going to determine the Gelfand-Kirillov dimension of the preprojective algebra $\Pi(\mathscr{T})$ of a modulated graph $\mathfrak{N}$ (see [4]).

THEOREM. The Gelfand-Kirillov dimension Dim $\Pi(\mathfrak{T})$ of the preprojective algebra $\Pi(\Re)$ of a modulated graph $\Re$ is equal to 0 if the graph defined by $\Re$ is Dynkin, 1 if it is Euclidean and $\infty$ otherwise.

REMARK. Thus, choosing a particular admissible orientation $\boldsymbol{\Omega}$ of $\mathfrak{T}$, $\operatorname{Dim} \Pi(\mathscr{N})=0$ if the respective tensor algebra $R(\mathscr{T}, \Omega)$ is of finite representation type, $\operatorname{Dim} \Pi(\mathfrak{N})=1$, if $R(\mathscr{N}, \Omega)$ is of tame representation type and $\operatorname{Dim} \Pi(\mathfrak{R})$ $=\infty$ otherwise (that is if $R(\mathscr{T}, \Omega)$ is of wild representation type).

PROOF OF THEOREM. For the calculation of the Gelfand-Kirillov dimension of $\Pi=\Pi(\mathfrak{T})$, we need a finite-dimensional $k$-subspace $V$ of $\Pi$ which generates $\Pi$. Choose an admissible orientation $\Omega$, and let $R=R(\Re, \Omega)$. Then $R$ is a finite-dimensional hereditary $k$-algebra, and $\Pi_{R}$ decomposes into the direct sum of all preprojective right $R$-modules, each occurring with multiplicity one:

$$
\Pi_{R}=\bigoplus_{t>0} \underset{1<s<n}{\bigoplus} P(s, t)
$$

see [4]. Let

$$
V^{m}=\underset{0<t<m}{\bigoplus} \bigoplus_{1<s<n} P(s, t) ;
$$

then $V^{0}$ can be identified with $R_{R}$ and we put $V=V^{1}$. It follows from [4] that

$$
V^{m+1}=V^{m} \cdot V^{1} \text { for all } m \text {. }
$$

[Namely, we can identify $P(s, t)$ with

$$
\underset{1<r<n}{\bigoplus} \operatorname{Hom}(P(r, 0), P(s, t)) \text {, }
$$

and in this way, we see that the product $P(s, t) P\left(s^{\prime}, t^{\prime}\right)$ is contained in $P\left(s^{\prime}, t+t^{\prime}\right)$ and that any element of $P\left(s^{\prime}, t+1\right)$ can be written as a sum of elements from the various products $P(s, t) P\left(s^{\prime}, 1\right)$ with $1<s<n$.] 
Let $f=\max _{1<s<n} \operatorname{dim}_{k} F_{s}$; here, $F_{s}$ are the division rings given in the modulation $\mathfrak{T}$. Thus, $f$ is the maximal $k$-dimension of a simple $R$-module, and therefore

$$
|\operatorname{dim} M| \leqslant \operatorname{dim}_{k} M \leqslant f|\operatorname{dim} M|
$$

for any $R$-module $M$. This shows that we may consider

$$
\varlimsup_{m \rightarrow \infty} \frac{\log \left|\operatorname{dim} V^{m}\right|}{\log m}
$$

instead of

$$
\varlimsup_{m \rightarrow \infty} \frac{\log \operatorname{dim}_{k} V^{m}}{\log m}
$$

in the definition of the Gelfand-Kirillov dimension. Now,

$$
\begin{aligned}
\left|\operatorname{dim} V^{m}\right| & =\sum_{t=0}^{m} \sum_{s=0}^{n}|\operatorname{dim} P(s, t)|=\sum_{t=0}^{m} \sum_{s=0}^{n}|\mathbf{p}(s, t)| \\
& \geqslant|\mathbf{p}(s, m)|=\left|c^{-m} \mathbf{p}(s, 0)\right| \quad \text { for any } 1<s<n .
\end{aligned}
$$

Now,

$$
\lim _{m \rightarrow \infty} \frac{\log |\mathbf{p}(s, m)|}{\log m}=\lim _{m \rightarrow \infty} \frac{m}{\log m} \cdot \frac{\log |\mathbf{p}(s, m)|}{m}=\infty
$$

since

$$
\lim _{m \rightarrow \infty} \frac{\log |\mathbf{p}(s, m)|}{m}=\log \rho
$$

by Proposition 2. Hence, if the graph defined by $\mathfrak{N}$ is neither Dynkin nor Euclidean, we see that $\operatorname{Dim} \Pi=\infty$.

In the case that the graph is Euclidean let $h$ be the standard homogeneous vector; thus $\mathbf{h}$ is the minimal positive integral vector stable under $c$. Furthermore, there is a natural number $u$ such that

$$
c^{u} \mathbf{x}=\mathbf{x}+\partial(\mathbf{x}) \mathbf{h}
$$

for some linear functional $\partial$ (called the defect); see [3]. In this case, we use the subspace $W=V^{u-1}$ in place of $V$; thus, $W^{m}=V^{m(u-1)}$. Let $d=\partial(\operatorname{dim} W)$. Then

$$
\operatorname{dim} W^{m}=\operatorname{dim} W+(m-1) d \mathbf{h},
$$

and therefore

$$
\operatorname{Dim} \Pi=\lim _{m \rightarrow \infty} \frac{\log \left|\operatorname{dim} W^{m}\right|}{\log m}=1 \text {, }
$$

because

$$
\lim _{m \rightarrow \infty} \frac{\log (m-1) d|\mathbf{h}|}{\log m}=1 .
$$

If the graph of $\mathfrak{T}$ is Dynkin, trivially Dim $\Pi=0$.

REMARK 1. In their paper, W. Borho and H. Kraft [2] define the superdimension DIM $A$ of an algebra $A$ as

$$
\varlimsup_{m \rightarrow \infty} \frac{\log \log \operatorname{dim}_{k} V^{m}}{\log m}
$$


It is easy to see that DIM $\Pi(\mathscr{N})=0$ if the graph defined by $\mathscr{N}$ is Dynkin or Euclidean, and that DIM $\Pi(\Re)=1$ otherwise.

REMARK 2. We also note the analogy to a theorem of V. Kac [9] which asserts that the Lie algebra associated to a Cartan matrix $C$ is of finite growth if and only if $C$ is Dynkin or Euclidean. However, whereas the theorem of Kac measures really the increase of the dimensions of the root spaces for the imaginary roots (all others have dimension 1), the theorem above measures the increase of the components of certain real roots with respect to a fixed basis of the root system.

\section{REFERENCES}

1. N. A'Campo. Sur les valeurs propres de la transformation de Coxeter, Invent. Math. 33 (1976), 61-67.

2. W. Borho und H. Kraft, Über die Gelfand-Kirillov-Dimension, Math. Ann. 220 (1976), 1-24.

3. V. Dlab and C. M. Ringel, Indecomposable representations of graphs and algebras, Mem. Amer. Math. Soc. No. 173 (1976).

4. ㄴ The preprojective algebra of a modulated graph, Proc. 2nd Internat. Conf. on Representations of Algebras (Ottawa 1979), Lecture Notes in Math., vol. 832, Springer, Berlin and New York, 1980, pp. 216-231.

5. I. M. Gelfand and A. A. Kirillov, Sur les corps liés aux algèbres enveloppantes des algèbres de Lie, Inst. Hautes Etudes Sci. Publ. Math. 31 (1966), 5-19.

6. C. M. Ringel, Algebras of wild representation type, Abstracts Conf. on Representations of Finite Dimensional Algebras, Oberwolfach 1977, pp. 95-102.

7. V. F. Subbotin and R. B. Stekolšcik, Jordan form of Coxeter transformations and applications to representations of finite graphs, Funkcional Anal. i Priložen 12 (1978), 84-85 = Functional Appl. 12 (1978), 67-68.

8. J. S. Vandergraft, Spectral properties of matrices which have invariant cones, SIAM J. Appl. Math. 16 (1968), 1208-1222.

9. V. G. Kac, Simple irreducible graded Lie algebras of finite growth, Math. USSR Izv. 2 (1968), $1271-1311$.

Department of Mathematics, Carleton University, Ottawa, Ontario k1S 5B6, Canada

Fakultät für Mathematik, Universträt Bielefeld, 4800 Bielefeld 1, West Germany 